\title{
Student interpretation of coefficients in Fourier series
}

\author{
Mikayla Mays and Michael Loverude \\ Department of Physics, California State University Fullerton, 800 N State College, Fullerton, CA, 92831
}

\begin{abstract}
As part of ongoing research in student use of math in upper-division physics, we examine student understanding of Fourier analysis. Fourier series are used in a variety of physics contexts and provide the first example of non-Euclidian vector spaces for many students. This study extends previous work in which we highlighted the procedural and conceptual difficulties students have when thinking about Fourier series. Using the concept image framework, as described by Tall and Vinner, we probe how students think about the constant coefficient in front of the Fourier series representation of a function and what it means when graphing the series. We also investigate whether students use the odd or even properties of a function to eliminate terms in the Fourier series. Data were collected at a large four-year university, including nine years of written data from an intermediatelevel Math Methods course as well as several interviews.
\end{abstract}

\section{INTRODUCTION}

As part of a larger study into how students use math in upper-division physics courses, we have investigated student understanding of Fourier analysis. For this paper we focus on the coefficients in a Fourier sine/cosine series. Fourier series are useful in a variety of physics contexts, including quantum mechanics, optics, and acoustics. Fourier analysis is a concept that can help students begin to make connections to the broader set of mathematical tools necessary to become effective physicists. Specifically, Fourier series can be connected to the idea of a vector space, where the harmonics of sine and cosine are the basis vectors and the coefficients are the magnitudes of each basis vector required to sum up to any periodic function. As a result, Fourier series are frequently covered in upper-division physics courses. Typical Fourier tasks in the canonical textbook for upper-division Math Methods courses [1] present students with a periodic function and ask them to generate a series representation; identifying terms and their associated coefficients.

Despite the importance of Fourier series, there has been little prior research on student understanding in this area. Jia et al [2] conducted 24 teaching-learning interviews with individual students taking an engineering course on linear systems that covered Fourier series. They described student difficulties in areas including 'properties of even and odd functions' and 'math-to-plot versus plot-to-math disconnect'. An optional question on Fourier series is included in the Colorado Classical Mechanics and Math Methods Instrument (CCMI) [3]. This current study extends our own previous work in which we highlighted the procedural and conceptual difficulties students have with Fourier series [4, 5].

\section{CONTEXT AND PRIOR RESULTS}

As noted, our work on student understanding of Fourier series has been ongoing. We briefly summarize previous results of our analysis of written problems to set the stage for the current data collection and analysis. The data that we initially analyzed comprised of seven semesters of post-
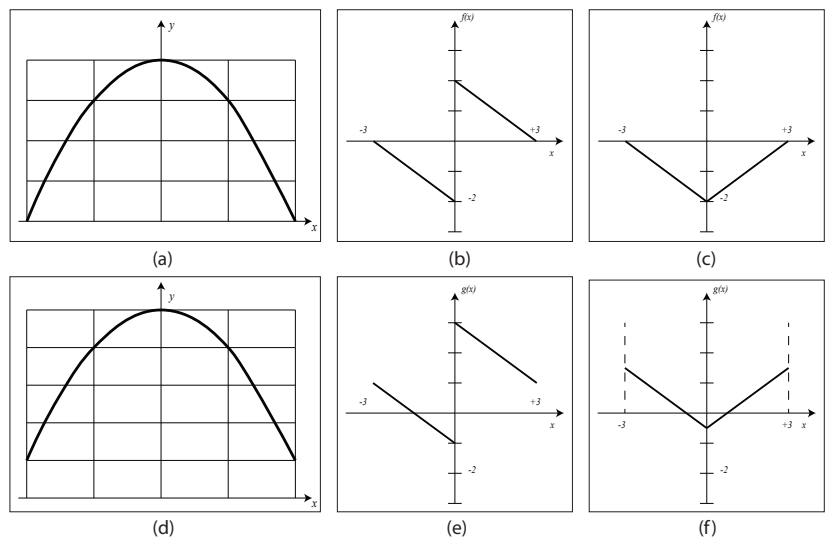

FIG. 1. Versions of the functions given on written assessments.

instruction graded assessments $(N=96)$ and five semesters of pre-instruction ungraded quizzes $(N=59)$. These were given in an upper-division Math Methods course using the text by Boas [1], taught by the second author of this paper. The course meets for two 75-minute blocks per week and has enrollment of between 12 and 24 students. Prerequisites include three semesters of calculus and at least two semesters of introductory physics. We collected data over eight years at California State University, Fullerton (CSUF), a large public university serving a diverse student population. Each written assessment had a series of questions but for this paper we focus on a subset; additional data are reported elsewhere [4].

On both the graded and ungraded assessments students were asked to determine whether $a_{0}$ and an arbitrarily-chosen coefficient ( $b_{3}$ for even functions or $a_{3}$ for odd functions) were positive, negative, or zero in the Fourier series for the periodic function shown in a graph that varied from semester to semester (see Fig. 1a-c). A final task on the graded assessments asked students which coefficients would change with a vertical shift of the function (see Fig. 1d-f).

To compute these coefficients the Math Methods textbook gives the following formulas [1]:

$$
a_{0}=\frac{1}{l} \int_{-l}^{l} f(x) d x
$$




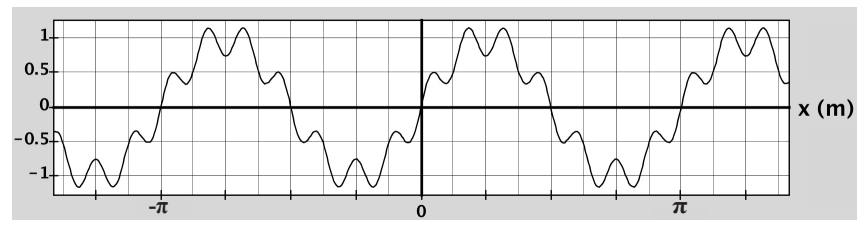

FIG. 2. Graph of the sum of two sine functions shown in interviews.

$$
\begin{aligned}
& a_{n}=\frac{1}{l} \int_{-l}^{l} f(x) \cos \left(\frac{n \pi x}{l}\right) d x \\
& b_{n}=\frac{1}{l} \int_{-l}^{l} f(x) \sin \left(\frac{n \pi x}{l}\right) d x
\end{aligned}
$$

When deciding if the $a_{0}$ coefficient is positive, negative, or zero students should realize that equation 1 will have the same sign as the area between the function and the $x$-axis. Thus $a_{0}$ is positive for Fig. 1a, zero for Fig. 1b, and negative for Fig. 1c. For $b_{3}$ or $a_{3}$, students were expected to reason with the symmetry of the function. For example, the Fourier series for an odd function will have only sine terms so the $a_{3}$ coefficient (which is associated with a cosine term) will be zero. For the question about the shifted function, students were expected to know that a vertical shift is mathematically characterized by the addition of a constant to the entire function, so the only coefficient to change is $a_{0}$.

Previous analysis of written data suggested that students were reasonably adept at setting up integrals that would allow them to determine the general form of the $a_{n}$ and $b_{n}$ coefficients of a Fourier series [4]. They were less successful on conceptual tasks. When asked whether the $a_{0}$ term is positive, negative, or zero for a given graph, about $75 \%$ of students were able to identify the correct sign both before and after instruction. However, student explanations suggest that this may overstate their understanding. Before instruction, approximately $25 \%$ of students used incorrect reasoning consistent with the idea that $a_{0}$ is associated with the $y$-intercept of the graph instead of the average value (compared to $14 \%$ after instruction). For all figures used (see Fig. 1a-c), this incorrect reasoning coincidentally gives the correct answers. Only about $23 \%$ of students (22 out of 96) correctly stated that only $a_{0}$ would change when the function is shifted vertically and gave the correct reasoning. Of those 22 students, 13 had also responded to the first question about $a_{0}$ correctly.

\section{INTERVIEWS}

The written data were suggestive of some patterns of reasoning, but left a number of questions unanswered. Explanations were often short and did not provide much insight into the response that $a_{0}$ is associated with the value of the function at $x=0$ or that a graph 'looked like a cosine.' Students mentioned other types of series, but it was not clear to what extent that influenced their responses. To probe these ideas, we conducted six one-on-one think-aloud interviews with undergraduate students who had completed the Math Methods course within the past three semesters. All six had the second author of this paper as their instructor. The course included the pre- and post-assessments from Fig. 1 and specific attention to the symmetry arguments used to discard coefficients.

For the interviews, we generated a graph of the sum of two sine functions with differing amplitudes and periods (see Fig. 2) using the PHET tutorial 'Fourier: Making Waves' [6]. Before Fourier series were explicitly mentioned, students were shown this graph and asked to express the function mathematically. This was included to be a conceptual analog to typical Fourier tasks that give a mathematical representation of the function and ask for the coefficients, since this gives a graph and asks for the symbolic representation.

The next tasks were developed in order to probe aspects of student thinking that seemed incomplete from the written data. We created a graph (see Fig. 3) that has a positive average value but a negative value at $x=0$ to eliminate the possibility that students could use incorrect reasoning to achieve the correct answer. We also created a graph of a triangle wave with an average value of zero so that we could ask if $a_{0}$ was positive, negative, or zero before and after we vertically shifted it (see Fig. 4). The function was created with even symmetry to probe the idea that even functions 'look like a cosine.'

\section{FRAMEWORK}

There are a number of different frameworks researchers have used to describe student thinking, including student difficulties, resources, and conceptual blending [7-9]. We conducted an initial analysis of the transcribed interviews that included describing and classifying student responses without a guiding framework and re-examining the data set for patterns [10]. This analysis suggested that individual students drew upon multiple, even contradictory, ideas when respond-

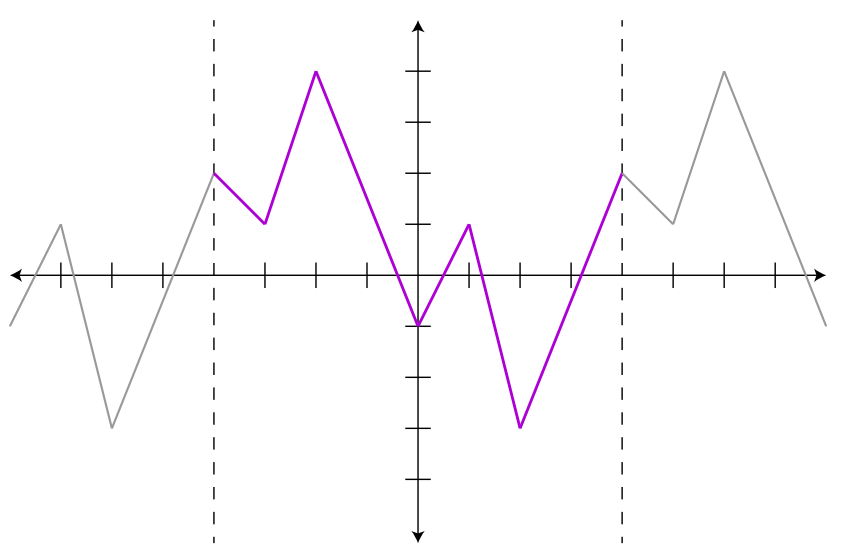

FIG. 3. Graph shown in interviews with a positive average value and a negative $y$-intercept. 


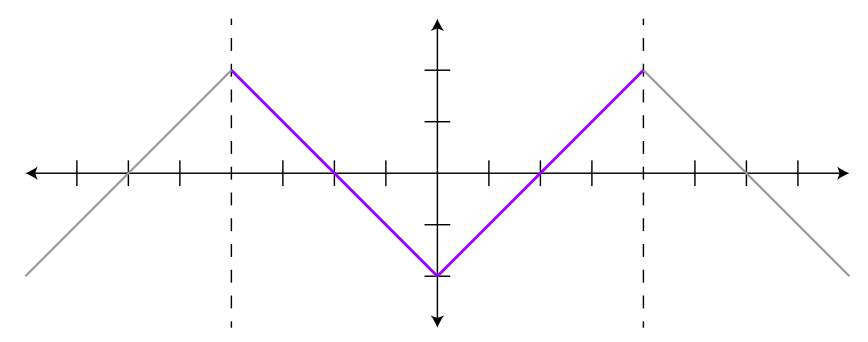

FIG. 4. Graph of the triangle wave shown in interviews.

ing to questions, which could be consistent with a resources analysis. However, the constituent ideas seemed to be more complex concepts that might themselves have structure. This larger grain size suggested the concept image framework.

In the concept image framework, as described by Tall and Vinner [11], a concept image is the entire cognitive structure that exists in a student's mind that relates to a particular concept. The evoked concept image is the element of the concept image elicited at a given time, possibly depending on context. This framework was developed to describe student reasoning in mathematics but has been adopted by several PER researchers [12-14] to describe student use of mathematics in upper-division physics. The concept image framework led us to focus on the elements that constitute students' concept image of a Fourier series.

\section{ANALYSIS}

The following were ideas that emerged in the responses of multiple students in our interview sample, such that we are confident in describing them as elements in the concept image evoked by the interview tasks. These elements can be incomplete and even contradictory, and lead to correct or incorrect answers. We do not claim that these represent a complete description of the concept image of any student and might expect that different tasks would evoke elements of the concept image that are not described here.

Intercept gives $a_{0}$ : Two of the six students used reasoning consistent with the idea that $a_{0}$ is associated with the value of the function at $x=0$. One of these students mentioned it very briefly when asked about the $a_{0}$ coefficient for the graph in Fig. 3, saying it was negative because, "I was looking at the origin and it was already dropped down," but he quickly doubted this and was unsure of the correct answer. The other student also said $a_{0}$ would be negative because, "The point on the $y$-axis is below the $x$-axis. So it's like the initial area is already negative." When asked to elaborate, he said, "Yeah $a_{0}$ kind of represents the initial... value of the function, so when $x$ is zero then it's whatever's on the $y$-axis so... $a_{0}$ exists when the rest of it doesn't exist, everything on either side of it, so because the function meets the $y$-axis below the, in the negative range, $a_{0}$ would be negative." This seemed to be consistent with some responses from the written data.
Area under the curve: For the purposes of this study, we use 'area under the curve' to mean the area between the function and the $x$-axis, which is a signed quantity depending on if it is above or below the $x$-axis. In all six interviews, students estimated the relative areas above and below the $x$-axis in order to determine whether there was more positive or negative area. Both students quoted in the 'intercept gives $a_{0}$ ' portion of this section changed their answers when the 'area under the curve' element of their concept image was evoked by later tasks. One was prompted to plug $n=0$ in to equation 2 (given to him earlier along with equation 3 ) and realized that it reduced to equation 1 . This led him to the conclusion that $a_{0}$ is positive for Fig. 3 because there is more positive area than negative area under the function. The other student changed his reasoning when he saw Fig. 4.

While this evoked concept image seemed to lead to correct answers, there is evidence that student understanding was incomplete. No student seemed to make the connection that adding a constant to a function is the same as adding that constant to each point on the graph, which offsets it from the $x$ axis. Moreover, three of the six students gave answers consistent with the notion that all of the terms in a Fourier series are related to areas. These same students, when asked about the coefficients that change when a function is vertically shifted upwards, answered that all of the non-zero coefficients would increase. This answer is the logical progression of the thought that each term is related to an area, as a vertical shift increases the area under the entire function. Tall and Vinner [11] suggest that a concept image can become restricted; the student uses only a portion of their concept image and may struggle when presented with a problem requiring a broader understanding of the subject. Schermerhorn [14] used the restricted concept image to describe student construction of differential elements for non-Cartesian coordinate systems, noting that "a restricted concept image can develop when students work for long periods repeatedly applying a given conceptual idea in a formulaic manner." We believe that the repeated use of integrals to determine Fourier coefficients has led to the formation of a restricted concept image in which area is associated with all coefficients.

Symmetry: A subsection of the Fourier series concept image that led students to productive reasoning was the idea of symmetry. Simplifying calculations using the symmetry of a system is a strategy often used to solve physics problems. For Fourier series, knowing the odd or even properties of a function allows you to discard certain coefficients without calculations. Students attended to multiple aspects of the graph, including symmetry but also some unintended features like the value at specific points. As in the written data, some students discarded coefficients based on their perception of whether the function 'looks like a cosine' but often did not articulate specific features of the graph in support of this. Two of six students spontaneously invoked a symmetry argument, however, the others quickly arrived at the right answer when prompted. At least one of the students we interviewed struggled to determine if a function was odd or even. 


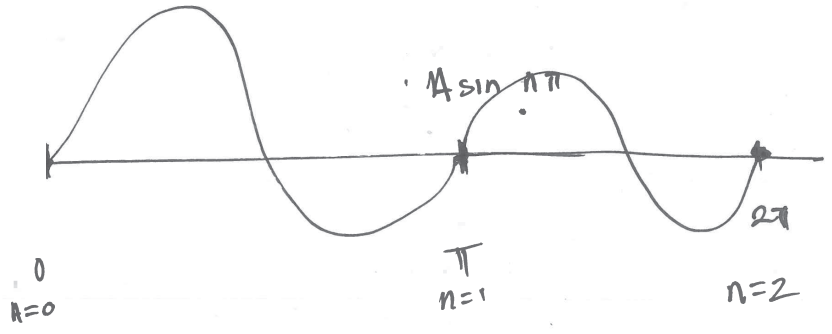

FIG. 5. Graph drawn by a student in an interview.

Index as spatial variable: Several student responses suggested an evoked concept image in which the index being summed over, $n$, was a spatial variable. While no student used this language, several responses indicated that they viewed $n$ as something that progressed along the $x$-axis. For example, one student said, "I believe this $a_{0}$ refers to the... the area of the graph when both of these are zero, basically when $n$ is zero and that means that this is the zeroth... I guess, period? And so the $n$ would be visible in seeing... which term's period you're looking at, so... this one [indicates central period of Fig. 3] is the zeroth and this one [indicates period to the right] would be... the first on either side [indicates period to the left] or the second going down." Another student drew a graph of a sine function and labeled the graph with $n=0$, $n=1$, and $n=2$ at intervals of $\pi$ (see Fig. 5).

\section{DISCUSSION}

The concept image framework seemed to be a productive way to analyze the interview data we collected. This framework allows for multiple contradictory elements to exist in the same concept image, which we saw in more than one interview. All of the students interviewed showed evidence of more than one of the elements of the Fourier series concept image listed in section $\mathrm{V}$.

In addition, we reflected on elements that were absent from students' concept images, such as the connection between Fourier series and vector spaces; no student spontaneously mentioned vector spaces. Student responses to the task involving the PHET graph (see Fig. 2) suggest that task in particular doesn't evoke elements of a Fourier series concept image for many students. Only two students spontaneously recognized that it related to Fourier series. Three others gave nested trigonometric functions (i.e. $\sin (\sin x)$ or similar) as their answer. This task also suggested that student understanding of Fourier analysis is closely tied to computation, as students struggled to complete it without formulas. This may further suggest that a restricted concept image has developed for some students, as described in the 'area under the curve' portion of section $\mathrm{V}$.

We saw evidence that some evoked concept images are tied to the symbolic form of the equations. The connection between all of the terms in the series and an 'area under the curve' seems to stem from the integrals in equations 2 and 3 . We believe this is due to the emphasis in introductory calculus courses on the 'area under the curve' definition of an integral. In addition, some students on written assessments seemed to rely on reasoning consistent with other types of series, such as Taylor series. We saw some indication of this in the interviews, but this element was only weakly evoked and seemed to be tied to the summation symbol in the general equation for Fourier series. It is possible that the graphical nature of our tasks didn't evoke this particular element for most students.

There were limitations to this study, as we still have some unanswered questions. We are still unsure what it means to students for a function to 'look like a cosine' and it is possible that other tasks and/or frameworks could answer these questions. In particular, there were parts of student reasoning that suggested it may be fruitful to consider conceptual blends.

\section{ACKNOWLEDGMENTS}

This work is supported in part by the National Science Foundation under Grant No. PHY\#1406035
[1] M. L. Boas, Mathematical Methods in the Physical Sciences, Wiley (2006), Chap. 7.

[2] C. Jia et al, in Proceedings of the 188th American Society for Engineering Education Conference, Vancouver, BC, 2011.

[3] M. D. Caballero et al, Phys. Rev. Spec. Top. - Phys. Educ. Res. 13, 010118 (2017).

[4] M. E. Loverude, in the 2011 PERC Proceedings, edited by N. S. Rebello, P. V. Engelhardt, and C. Singh.

[5] https://www.dropbox.com/s/1963tp7yv19zgd4/ MM2017PERC.pptx. Retrieved 7/3/2018.

[6] https://phet.colorado.edu/en/simulation/fourier. Retrieved $7 / 3 / 2018$

[7] P. R. L. Heron, in Enrico Fermi Summer School on Physics
Education Research, Varenna, Italy (Italian Physical Society, 2003).

[8] D. Hammer, Am. J. Phys. 68, S52 (2000).

[9] G. Fauconnier and M. Turner, The Way We Think: Conceptual Blending and the Mind's Hidden Complexities, (Perseus Books Group, New York, 2002).

[10] J. Corbin and A. Strauss, Qualitative Sociology 13, 1 (1990).

[11] D. Tall and S. Vinner, Educ. Stud. Math. 12, 151 (1981).

[12] L. Doughty, E. McLoughlin, and P. van Kampen, Am. J. Phys. 82, 1093 (2014).

[13] L. Bollen, P. van Kampen, and M. De Cock, Phys. Rev. Spec. Top. - Phys. Educ. Res. 11, 020129 (2015).

[14] B. P. Schermerhorn, Ph.D. thesis, University of Maine, 2018. 